\title{
REVIEW
}

\section{Multiple effects of grape seed polyphenolics to prevent metabolic diseases}

\author{
Torey ARVIK ${ }^{1}$, Hyunsook KIM ${ }^{2}$, James SEIBER ${ }^{3}$, Wallace YOKOYAMA $\left.(\bowtie)\right)^{4}$ \\ 1 Sonomaceuticals/WholeVine Product LLC, Santa Rosa, CA 95403, USA \\ 2 Hanyang University, Seoul 04763, Korea \\ 3 Department of Food Science, University of California, Davis, CA 95616, USA \\ 4 United States Department of Agriculture (USDA), Agricultural Research Service (ARS), Western Regional Research Center, \\ Albany, CA 94710, USA
}

\begin{abstract}
Obesity is increasing in developing countries. Population studies show a relationship between affluence and obesity. Changing food intake patterns with affluence such as preference for foods with less astringent polyphenolic compounds and dietary fibers may increase risk of metabolic dysfunctions due to caloric imbalance. Animal models of obesity consistently show that grape seed procyanidins prevent increases in body and abdominal adipose weight gain, plasma cholesterol, liver weight gain and inflammation in animals on high fat diets. The mechanisms are not clear because the oral intake of procyanidins results in pleiotropic interactions with proteins in the mouth, stomach, small intestine, cecum and colon that affect the rate of digestion of bioavailability of macronutrients, sterols, and dietary fiber. Procyanidins also bind bile acids and reduce intestinal permeability to inflammatory bacterial cell wall fragment. Procyanidins are not degraded or metabolized until reaching the lower gut where they can be metabolized into phenolic acids by gut bacteria. While they are metabolized by gut bacteria, they also alter total numbers and distribution of phyla and species of gut bacteria. Gut bacteria are recognized as significant contributors to obesity and obesity related metabolic diseases. The review examines the different pleiotropic effects of grape seed procyanidins that have a significant effect on metabolic disease in animal models of obesity.
\end{abstract}

Keywords grape seed, obesity, procyanidins, high fat, microbiota, animal models

Received October 13, 2017; accepted June 12, 2018

Correspondence: Wally.Yokoyama@ars.usda.gov

\section{Introduction}

In recent years research has identified low grade inflammation accompanying obesity as necessary for the development of chronic metabolic diseases ${ }^{[1,2]}$. The low grade inflammation is a reaction primarily by adipocytes and macrophages to cell wall fragments of Gram-negative bacteria (lipopolysaccharides, LPS) passing through the intestinal wall into the body ${ }^{[3-5]}$. This form of inflammation has a dietary connection and diet is related to the affluence of the individual. The epidemic of obesity is a serious global public health concern because it is associated with chronic metabolic diseases ${ }^{[6]}$ diabetes, cardiovascular disease, stroke and some cancers. These chronic diseases incur costly treatments to individuals and government health services, and result in long-term disabilities in later life that at the least reduce life satisfaction and more often extended periods of pain before a shortened life.

At the molecular level, LPS, the initiators of an inflammation cascade, are the root cause of obesity. The consumption of the typical diets of affluent Western countries rather than traditional diets and the decrease in energy expenditure as countries become more affluent ${ }^{[7]}$ are accelerating LPS based inflammation. High fat diets increase the secretion of bile acids necessary for their digestion but high fat and bile acids change the intestinal microbiota and increase intestinal permeability to LPS. Studies have shown that as the population of middle income increases, obesity increases. In addition to energy imbalance, higher dietary fat content increases bile acid secretion into the intestinal lumen that may increase gut permeability to LPS and alter the gut microbiome ${ }^{[8]}$. Since World War II, the USA has had an expanding economy and is one of the most affluent countries in the world. It also has one of the highest rates of obesity, currently about $37 \%$. The per capita income in China was 385, 7942, and 


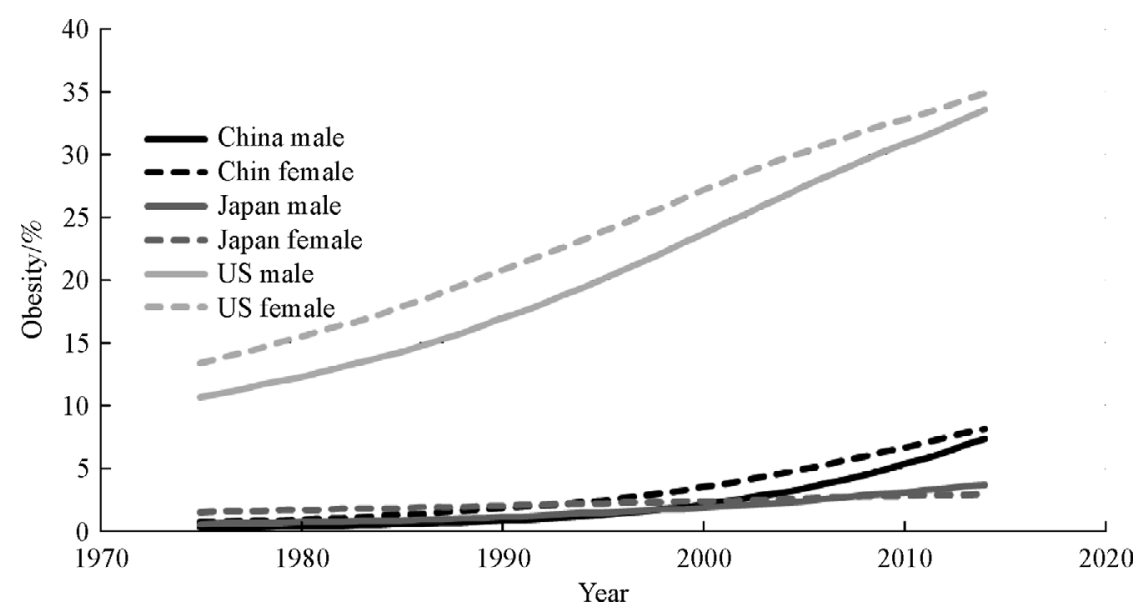

Fig. 1 Percent obesity in US, China and Japan from 1975 to 2014. The percentage of obese persons increased in China. The per capita income increased from 385 to 49992 CNY between 1978 and 2015.

49992 CNY in 1978, 2000, and 2015, respectively (China Statistical Yearbook 2016). The rapid economic development and affluence in China has increased the rate of obesity (Fig. 1) from $2.1 \%$ in 2000 to $7.4 \%$ in 2014. Diabetes is often a consequence of obesity and in China the number of diabetics is expected to increase to over 37 million by 2025 , the highest of any country in the world other than India ${ }^{[9]}$. In contrast, the economy of Japan has been lackluster in recent decades and obesity in Japan has remained low, which supports the relationship between diet, affluence and obesity.

Also, diabetes has a genetic component (Fig. 2). While the level of obesity in China is relatively low compared to the USA, the effects of affluence on the genetic background of the Chinese population is seen in the higher rate of obesity of Chinese males and females than US males and females. Other non-caucasian races, such as Hispanics and Africans, also have greater risk of diabetes. While diet can express the genetic potential for diabetes the low rate of diabetes in Japanese females for the past 40 years, $1.5 \%-3.0 \%$, reinforces the link between diet, obesity and diabetes.

Preventing obesity or reducing excess weight is extremely difficult and dietary restriction, drugs and supplements have not been successful in reducing individual and population excess weight and obesity despite decades of research.

Diet has a significant role in the health and disease status of individuals. High fat diets facilitate inflammation but some food components such as dietary fiber and polyphenolics that reach the gut may prevent or moderate weight gain, abdominal adipose weight, increases in blood

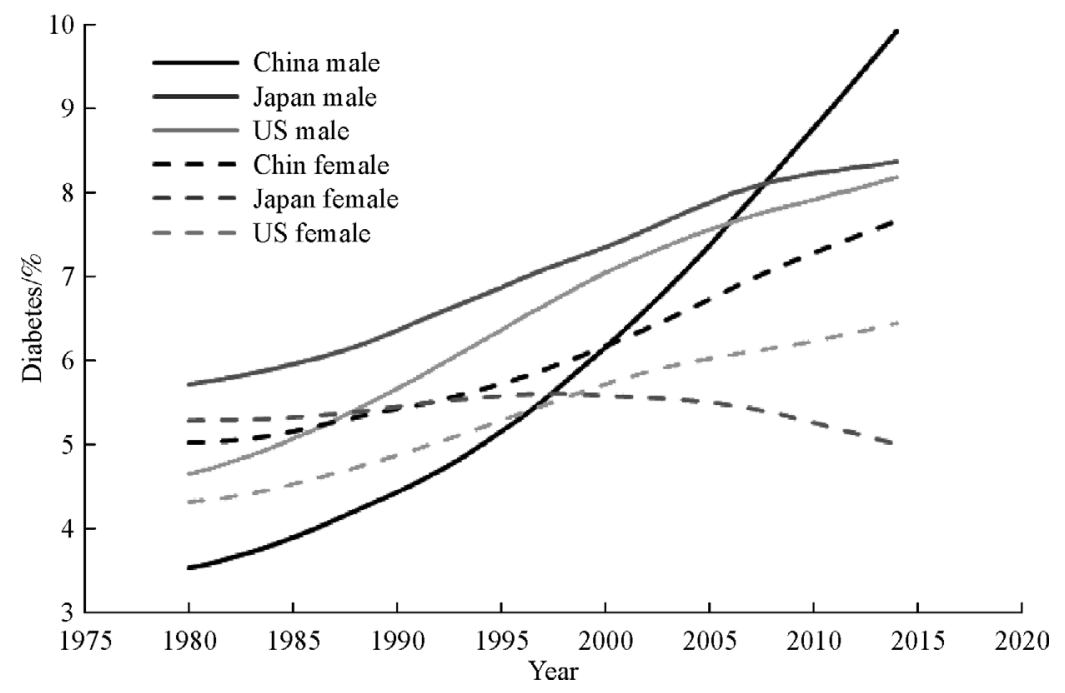

Fig. 2 The percentage of male diabetics in China has almost doubled in the past 20 years. A combination of increasing prosperity and genetics play major roles. Japanese males, US males and US females, but not Japanese women, have increased rates of diabetes in the same period ${ }^{[10]}$. 
lipids, fatty liver and inflammation in animal models on high fat diets. These components are non-nutritive and mainly indigestible but are metabolized in the lower gut by bacteria, yeast and other organisms (microbiome). The intake of dietary fiber and polyphenolic compounds has been shown to reduce risk factors for diabetes, cardiovascular disease and other chronic diseases by mechanisms related to fecal excretion of fat, cholesterol, and bile acids, and reduction of chronic inflammation. Polyphenols occur with dietary fibers in the outer layers of fruits and vegetables and in seeds. Intake of vegetables has decreased in China since $1991^{[11,12]}$. Over the past decade research has focused on chronic low grade inflammation as a result of interactions between diet and gut microbiome ${ }^{[2,3,5,6]}$. As populations become more affluent, dietary fiber, associated phenolic compounds and polyphenolics are processed away from plant foods to make them more palatable. For example, cereal brans are a major source of non-starch polysaccharide dietary fibers and phenolic, but consumers prefer the white endosperm and the bran is milled away to improve consumer acceptance. Fruit and vegetable skins, and seeds contain high concentrations of polyphenolics. The focus of this review article is on a subgroup of polyphenolics: procyanidins, oligomers of catechin and epicatechin, because there has been a significant amount of research on this group of plant compounds which may be a model for understanding nonabsorbable plant phytochemicals.

Procyanidins have pleiotropic effects in the digestive system. Before reaching the gut, procyanidins interact with salivary proteins in the mouth and other proteins such as proteases, lipases, esterases, amylases and many other digestive enzymes in the stomach and small intestine. As a result the rate of digestion or the complete digestion of proteins, carbohydrates, cholesterol esters, bile acid conjugates, and triglycerides may be affected. Another pleiotropic effect of procyanidins is their ability to bind bile acids ${ }^{[13]}$, as well as alter their deconjugation, affecting their uptake, binding to intestinal farnesoid $\mathrm{X}$ receptor (FXR) and synthesis of tight junction protein. After reaching the cecum and colon, procyanidins are metabolized into small molecules that are absorbed into the body and may alter metabolism and inflammatory activity in intestine, liver, adipose, brain and other organs. Given the interactions occurring in the mouth and digestive system, this review focuses on studies of oral intake in animal, and human studies of obesity and related chronic diseases.

\section{Composition and chemistry}

Procyanidins in grapes are found mainly in skins and seeds, but almost all of the physiological and nutritional effects in animal models and humans have used grape seed extracts. The most prominent components of grape seed extracts, the proanthocyanidins (PAs) that include their oligomers, the procyanidins, have shown to be the most physiologically active components. PAs are found in apples, pine bark, cinnamon, aronia berries, cocoa beans, blueberry and other edible berries, nuts, green tea, and potato skins as well as in grapes ${ }^{[14]}$. Plant polyphenol extracts, mainly PAs, are a mixture of monomers, $(+)$-catechin (CAT), (-)-epicatechin (EPI), and their procyanidin oligomers, and gallic acid esters ${ }^{[15]}$. A range of semiquantitative methods that report total PAs expressed as gallic acid or (epi)catechin equivalents are typically used to characterize grape seed PA extracts. While these analytical methods provide a single number for total polyphenolics, there is a great deal of ambiguity in the oligomeric composition of grape seed extracts in published research $^{[16,17]}$ (Fig. 3).

The oligomeric composition is important because some studies have shown that there is an optimum size for highest bioactivity. PA oligomeric composition and amounts change with harvest date. PAs in grape seeds are concentrated in the outer layers (outer integument) as shown by p-dimethylaminocinnamaldehyde, a PA-specific stain $^{[19]}$. The amounts and degree of polymerization of procyanidins decreases rapidly after the grapes start to ripen (veraison). For example, 2 weeks after reaching veraison the mass of extracted procyanidins in Canadian Shiraz berries decreased by about $40 \%{ }^{[20,21]}$. Drying and condensation of the seed during maturity may also reduce extractability. The mean degree of polymerization (mDP) also decreases rapidly during the early stages of veraison. In the Canadian Shiraz study, mDP decreased from about mDP 9 to 6 in the first 6 weeks. Analysis of the changes in $\mathrm{mDP}$ by thiolysis and HPLC suggest that rather than degradation the larger procyanidins may be covalently attached to larger molecules, such as proteins, or are oxidized, reducing the overall $\mathrm{mDP}$.

Grape cultivar, environment ${ }^{[22,23]}$ and cultural practices affect total seed polyphenol content and distribution of procyanidin oligomers. Generally, the concentration of the monomers are higher than oligomers as shown in Fig. 4 for four red and two white grape cultivars ${ }^{[15]}$. Pinot Noir and Chardonnay contain the highest amounts of flavanols in the red and white grape cultivars, respectively. After winemaking, the concentrations of flavanols in pomace from red grapes may be lower since the seeds and skins of red grapes are fermented during winemaking and a significant amount of polyphenols, particularly the more soluble monomers, are extracted into the wine. Chardonnay seeds are often used as the source of polyphenols in nutrition studies because of its high content.

\section{Pleiotropic interactions in the gut lumen and bioavailability}

Grape PAs, known as tannins in the wine industry, are the cause of astringency in wine. PAs bind to proteins, and in 


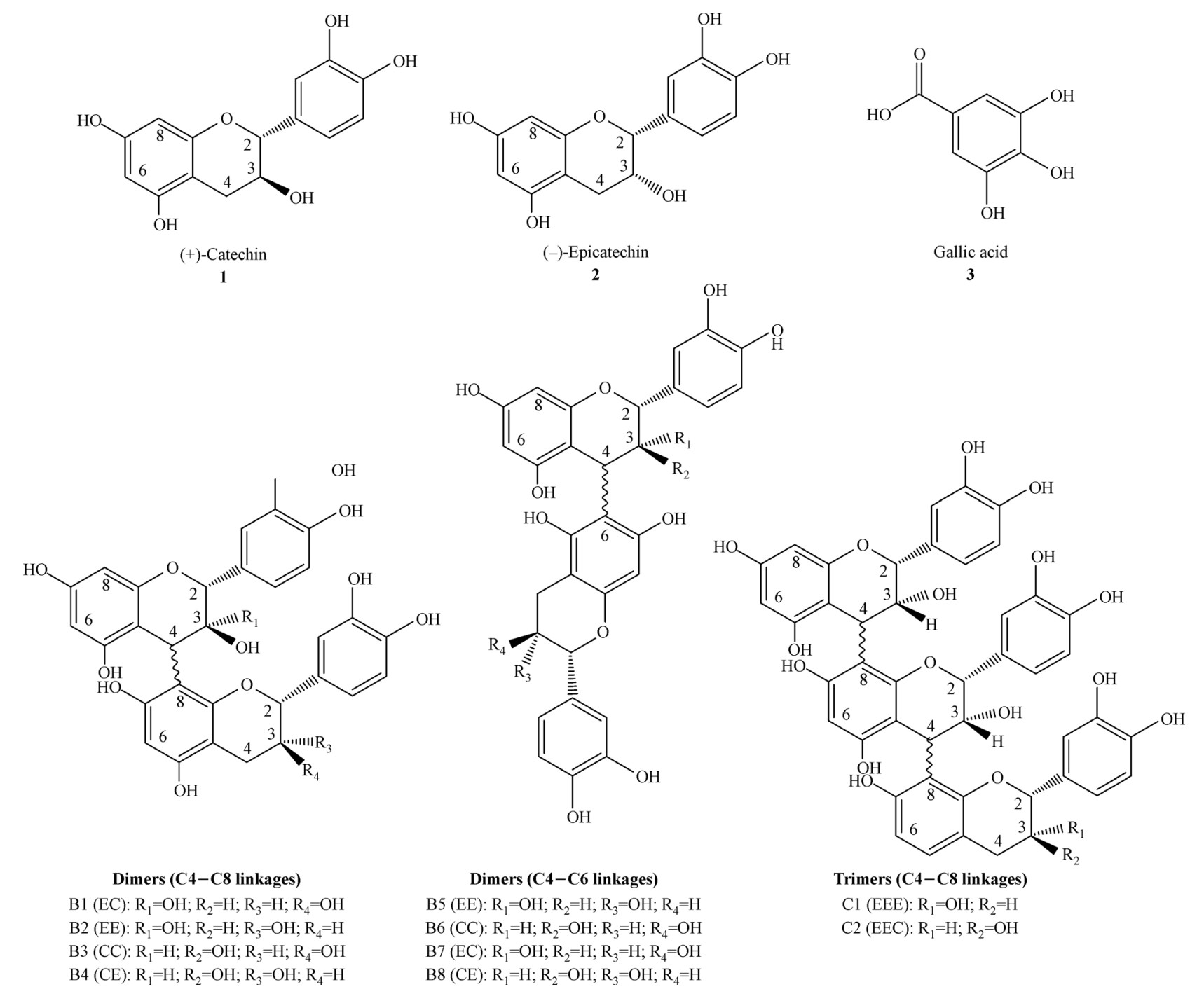

Fig. 3 Structures of monomers, catechin and epicatechin, and their oligomers (B-Type ${ }^{[18]}$

the mouth, they bind to proline rich proteins (PRP) salivary proteins that are induced by PAs and these PRP appear to have evolved to bind tannins. Colloidal suspensions are formed by tannin binding below the critical micelle concentration of tannin. Proteins bind at specific sites and form a colloidal suspension, whereas above the critical micelle concentration, the binding is nonspecific and forms precipitates $^{[24]}$. PRP may be secreted by the parotid and submandibular salivary glands in order to overcome the unpleasant taste of the seed or fruit, or are induced to spare digestive enzymes, which facilitates hydrolysis and absorption of macronutrients (fat, carbohydrates, and amino acids). Chronic intake of grape seed tannins has been shown to result in hypertrophy of the parotid and submandibular glands, suggesting that this is important for the survival of animals feeding on tannin rich plants ${ }^{[25]}$. The salivary gland secretes acidic, basic and glycosylated proteins, and tannins bind preferentially to the acidic group ${ }^{[26,27]}$. Pectin and other polysaccharides interfere with the binding of tannins to salivary proteins ${ }^{[27]}$ and trypsin $^{[28]}$.

PAs have diverse effects on intestinal enzymes. In the small intestine ( + )-CAT and (-)-EPI are absorbed ${ }^{[29]}$, but oligomeric procyanidins are not readily absorbed ${ }^{[30]}$, and may inhibit digestive enzymes and modulate energy, cholesterol and bile acid metabolism. In one of the earliest studies of grape tannins in rats, Glick and Joslyn ${ }^{[31]}$ showed that grape seed tannins increased excretion of fecal nitrogen to $24 \%$ in rats fed either $5 \%$ grape seed tannins or $5 \%$ tannic acid, compared to $6 \%$ in control rats. Catechin and gallic acid had no effect on protein excretion. The amino acid composition of the excreted proteins was different from dietary casein, and suggested that they were derived from endogenous enzymes. Possibly the binding of enzymes by tannins required the secretion of increased amounts of enzymes. In vitro, the proteolysis of BSA by pepsin and chymotrypsin decreased with increased tannin (900-3000 Da), but tannins increased proteolysis by 


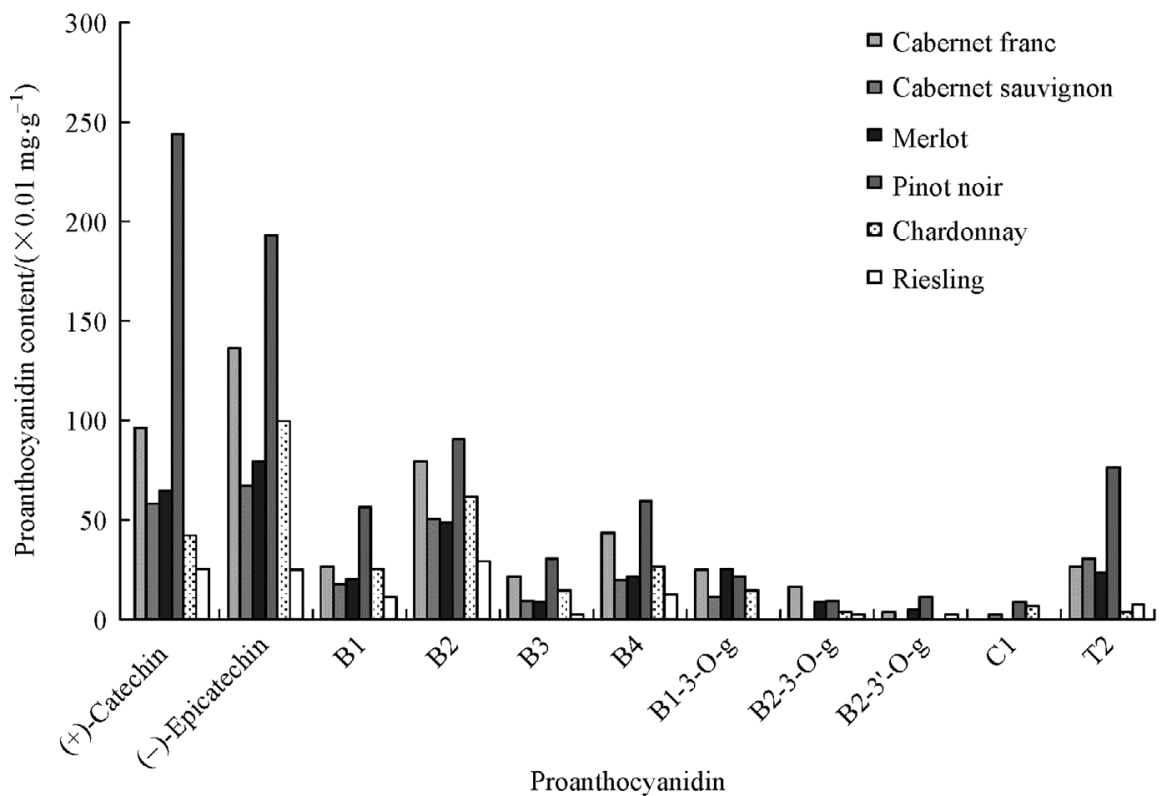

Fig. 4 Proanthocyanidin monomers, oligomers, and gallates. The red grapes are generally higher in proanthocyanidins (PAs). Chardonnay has a higher content of PAs than the other white grape Riesling. After processing into wine, Chardonnay grape seeds often have higher PA content because white grape seeds are not fermented with the juice and the PAs are not extracted. Alcohol extracts of Chardonnay seeds are often used in human and animal studies. Catechin and epicatechin are monomers. B1-B4 are dimers and Bx-3-O-g are dimer gallates. $\mathrm{C} 1$ and $\mathrm{T} 2$ are trimers.

trypsin ${ }^{[32]}$. Denaturation of BSA by tannins was suggested as the mechanism behind the increased trypsin digestion.

Intestinal alkaline phosphatase (iAP) is induced by LPS and other microbial components and has important antiinflammatory functions. Intestinal alkaline phosphatase detoxifies lipopolysaccharides (LPS) and modulates the microbiota homeostasis ${ }^{[33-35]}$. Sucrase activity and iAP in the mid-jejunum was reduced by about a third and two thirds, respectively, in rats fed grape tannins for $31 \mathrm{~d}^{[36,37]}$. However, tannin had no effect in the duodenum. In vitro experiments showed that pancreatic biliary juice reversed the inhibitory effects of tannins and suggested that the reabsorption of bile acids further down the intestine restored tannin binding and inhibition. Dietary iAP reversed a metabolic syndrome in mice fed high fat $\operatorname{diets}^{[38]}$. Therefore, inhibition of iAP is paradoxical since obesity and related chronic metabolic diseases are associated with LPS-induced inflammation which suggests that grape PAs in high fat diets inducing higher bile acids may not inhibit iAP.

Apple procyanidins consist of monomeric and oligomeric (epi) catechins similar to grape procyanidins. Apple procyanidins at $1 \mathrm{~g} \cdot \mathrm{kg}^{-1}$ bodyweight, reduced postprandial triglycerides in humans when ingested with corn oil ${ }^{[39]}$. In vitro, the same investigators found that pentamers and higher oligomers were the most effective inhibitors of pancreatic lipase, whereas monomers and dimers had little inhibitory activity. In vitro, grape seed extract has also been shown to decrease human pancreatic lipase activity in a dose dependent manner ${ }^{[40]}$. In vitro cholesterol esterase activity decreased by $70 \%$ with grape seed extract at $50 \mu \mathrm{g} \cdot \mathrm{mL}^{-1}$ and pancreatic lipase activity decreased by $75 \%$ with grape seed extract at $4.75 \mathrm{mg} \cdot \mathrm{mL}^{-1[41]}$. In the same study, grape seed extract and cholestyramine at $1 \mathrm{mg} \cdot \mathrm{mL}^{-1}$ bound taurocholic acid and glycodeoxycholic acid, and inhibited the formation of cholesterol micelles. Plasma triglycerides were also decreased in a dose response manner in rats in the following $10 \mathrm{~h}$ after gavage with olive oil and grape seed extract.

Prior to the discovery of the importance of gut microbiome to health, and their ability to metabolize procyanidins and alter microbiome distribution due to procyanidin feeding, the negligible or low bioavailability of procyanidins made it difficult to explain their physiological effects. Bioavailability is typically determined by measuring the analyte in blood or urine. In 2002, Donovan et al. ${ }^{[42]}$ showed that catechin, but not procyanidin $B_{3}$ (catechin- $(4 \alpha \rightarrow 8)-$ catechin) or procyanidin, in grape seed extracts were measurable in blood or urine. The measurements were made in rats fed $20 \mathrm{mg}$ of (+)-catechin, or 200 (GS1) or $400 \mathrm{mg}$ (GS2) of a grape PA mixture and killed after 3,9 and $24 \mathrm{~h}$. GS1 contained $20 \mathrm{mg}$ catechin, $14 \mathrm{mg}$ epicatechin, $11 \mathrm{mg}$ procyanidin $\mathrm{B}_{1}$, $7 \mathrm{mg}$ procyanidin $\mathrm{B}_{2}$ and $3 \mathrm{mg}$ procyanidin $\mathrm{B}_{3}$. GS2 contained twice the amounts of GS1. Blood and urine were analyzed by HPLC using colorimetric detection. Urinary catechin recovery from the catechin group was $37 \%$, and $43 \%$ and $32 \%$ from the 200 and $400 \mathrm{mg}$ grape seed extract 
groups, respectively. Most, 54\%-69\%, of the recovered catechins were the 3'-O-methylcatechin. (-)-Epicatechin was also recovered from the grape seed extract groups at $40 \%$ and $29 \%$ for the 200 and $400 \mathrm{mg}$ dose groups, respectively. Procyanidins $\mathrm{B}_{1}, \mathrm{~B}_{2}$ and $\mathrm{B}_{3}$ fed to the GS1 and GS2 groups were not detected in urine. Neither catechin nor epicatechin or their 3'-O-methylated products were found in blood from rats fed catechin, procyanidin $\mathrm{B}_{3}, \mathrm{GS} 1$ or GS2. These results suggest that metabolism and absorption of procyanidins is neglible or does not occur. In the same year, Baba et al. ${ }^{[43]}$ reported the absorption and urinary excretion of procyanidin $B_{2}$ (epicatechin- $(4 \beta \rightarrow$ 8)-epicatechin) in rats by a more sensitive LC-MS. Procyanidin $B_{2}$ was found in urine but the amount was estimated to be $0.34 \%$ of the oral dose. Procyanidin $\mathrm{B}_{2}$ was also found to increase in blood, peaking at $30 \mathrm{~min}$ after ingestion, but the amount relative to oral dose was not stated.

Although bioavailability of PAs of intact flavonoids by urine and blood analysis appeared to be negligible, PAs are metabolized by gut microbes into phenolic acids. In 2013, Choy et al. ${ }^{[44]}$ showed that lower amounts of PA monomer and oligomers were extracted from the colon and feces compared to amounts fed suggesting some remained in the animal. Rats were fed a semisynthetic diet containing $0.25 \%$ grape seed extract (GSE) for $10 \mathrm{~d}$. The GSE contained $12 \%, 14 \%, 19 \%, 6.4 \%$, and $4.0 \%$ monomers, dimers, trimers, tetramers, and pentamers respectively, and lower amounts of higher oligomers. The ratio of monomers, dimers, trimers, tetramers and pentamers in the feces after $10 \mathrm{~d}$ of GSE feeding was about 1:2:5:7:5 compared to 1:1:1.6:0.5:0.3 of the GSE ingredient. The results show that the monomers were absorbed and higher excretion of trimers and tetramers compared to other oligomers. By averaging intake and excretion of five rats, an estimate of mass balance showed that $1.7 \%$ of monomers were excreted. Excretion of dimers and trimers were $3.9 \%$ and $6.8 \%$, while tetramers, pentamers and hexamers were $27 \%$, $29 \%$ and $15 \%$, respectively. It must be noted that rats are coprophagic and that the fecal polyphenolic compounds may be recycled through the rat decreasing the amount of excreted PAs. In 2014, the same group analyzed the PAs and PA metabolite content in the feces from female pigs fed $\mathrm{GSE}^{[45]}$. The pigs were fed a normal diet containing $1 \%$ GSE for $6 \mathrm{~d}$ and feces were collected daily. Dimers, trimers, tetramers and pentamers were found at about $100 \mu \mathrm{g} \cdot \mathrm{mL}^{-1}$. Catechin and epicatechin were about 5 and $8 \mu \mathrm{g} \cdot \mathrm{mL}^{-1}$. The major metabolites were 4-hydroxyphenylvaleric acid and 3-hydroxybenzoic acid.

The absence of gut bacteria to the anti-obesity effects of grape seed PAs were shown recently in a mouse study by Liu et al. ${ }^{[46]}$. C57BL/6 mice on high fat diets were administered grape seed polyphenol extract (GSPE, $300 \mathrm{mg} \cdot \mathrm{kg}^{-1} \mathrm{bw}$ ) by gavage. Grape seed treatment resulted in lower levels of plasma inflammatory cytokines $\mathrm{TNF} \alpha$ and IL-6, and reduced epididymal fat. There was a difference in diversity of gut bacteria and the phylum Proteobacteria increased in mice treated with GSPE. Treatment with ampicillin and neomycin resulted in similar bacteria populations in both HF control and HF GSPE treated groups. Antibiotic treatment also abolished differences in epididymal adipose weight and plasma inflammatory cytokines. Dietary fat composition has a major effect on microbiota composition. On high fat diets, Firmicutes predominated in the cecum of rats ${ }^{[47]}$. On a control diet the ratio of Firmicutes to Bacteroidetes was $5: 3$, but the addition of $1.25 \mathrm{mmol} \cdot \mathrm{kg}^{-1}$ diet cholic acid resulted in predominately (99\%) Firmicutes population. Cholic acid is rapidly converted to deoxycholic acid in the gut by bacteria. In vitro studies of cecal bacteria showed that the half maximal inhibitory concentration $\left(\mathrm{IC}_{50}\right)$ of deoxycholic acid was lower than cholic acid and more inhibitory to Bacteroidetes than Firmicutes. The researchers suggested that the effect of high fat diets in promoting Firmicutes is due to the increased secretion of bile acids to digest the additional fat. PAs may also inhibit bacterial survival directly. In the presence of a PA trimer, epicatechin- $(4 \beta \rightarrow 6)$-epicatechin- $(2 \beta \rightarrow \mathrm{O} \rightarrow 7,4 \beta$ $\rightarrow$ 8)-catechin, the growth of Bacillus cereus, a Grampositive bacteria, was inhibited ${ }^{[48]}$. The investigators found that the over 500 genes related to normal function of the cell membrane and wall of $B$. cereus were upregulated and glucose absorption was impaired compared to controls.

\section{Grape proanthocyanidins, obesity and metabolic dysfunction in animal models and humans}

Many highly effective nutraceuticals, such as curcumin, resveratrol and grape seed procyanidins, are not readily absorbed into the body so that mechanisms based on absorption of the intact molecule and subsequent action in target organs should be considered carefully. As discussed previously, grape seed PAs are a mixture of absorbable monomers and nonabsorbable oligomers. In possibly the earliest study of grape seed polyphenols in an animal model of hypercholesterolemia and weight gain, Tebib et al. ${ }^{[36]}$ showed that oligomeric procyanidins prevented the physiological characteristics of metabolic diseases more effectively than a monomeric fraction in rats on high fat diets ${ }^{[49]}$. In their study rats were fed a high fat diet (17\% highly saturated fat) supplemented with either $1 \%$ of a monomeric fraction or $1 \%$ oligomeric (dimers to tetramers). Although feed intake between control, monomeric supplemented and oligomeric supplemented did not differ, weight gain and body fat of the rats fed the monomer and oligomers were $19 \%$ and $53 \%$ lower, respectively, compared to the controls. Total and LDL cholesterol of the oligomeric fed group were about $65 \%$ and $75 \%$ lower, respectively, compared to the controls. Fecal cholesterol and bile acid excretion were about twice and four times 
higher, respectively, in the oligomer fed rats, compared to the controls. In 1999, Martin-Carron et al. ${ }^{[50]}$ reported that rats on a diet supplemented with $15 \%$ lard, a grape pomace fiber and polyphenol product for 4 weeks had reduced plasma cholesterol, but weight gains did not differ. The fiber-polyphenol ingredient contained $36.6 \%$ condensed tannins, $1.3 \%$ soluble polyphenols and $47.7 \%$ insoluble dietary fiber. The researchers concluded that bile acid homeostasis, requiring synthesis of bile acid from cholesterol and movement of cholesterol from blood to liver, was responsible for the lowering of plasma cholesterol.

In rats fed low-fat lithogenic diets (1\% cholesterol), plasma and liver cholesterol did not differ after administration of $0.1-1.0 \mathrm{~g} \cdot \mathrm{kg}^{-1}$ bodyweight GSE compared to the controls $^{[51]}$. In this study, weight gain and feed intake decreased with increasing dose of grape seed in animals administered GSE on either lithogenic or normal diets, compared to the controls. Excretion of some bile acids, particularly lithocholic acid, increased in feces with increasing GSE dose. Many in vitro studies have been reported, but these are not reviewed because of their focus on intestinal lumen and microbiome interactions ${ }^{[52-54]}$.

More recently, the attenuation of obesity induced inflammation by grape seed PAs were demonstrated by molecular methods. Décordé et al. ${ }^{[55]}$ determined the expression of adipocytokines in hamsters on high fat diets supplemented with Chardonnay grape seed extracts for 12 weeks. The extract was reported to contain $448 \mathrm{mg} \cdot \mathrm{g}^{-1}$ polymeric tannins as leucocyanidin equivalents. Hamsters on high fat, hypercholesterolemic diets are often preferred for studies of cholesterol metabolism because they have identical primary bile acids as humans, low rate of hepatic cholesterol synthesis in males similar to humans, also a gallbladder is not present in rats, and rats are deficient in cholesteryl ester transfer protein ${ }^{[56]}$. Hamsters on a high fat diet, had leptin increased by $73 \%$ and adiponectin decreased by $54 \%$ compared to those on a low fat diet. Less weight gain and abdominal body fat were observed in the GSE fed hamsters supporting the earlier rat study. Blood glucose was also lower, but insulin levels were similar to the controls. The plasma adipocytokines, leptin and adiponectin, determined by Western blotting were lower and higher, respectively, in GSE fed hamsters and the controls. Elevated plasma leptin and decreased adiponectin are characteristics of obesity and type 2 diabetics $^{[57]}$. Leptin regulates appetite and the innate and adaptive immune system ${ }^{[58]}$. Adiponectin increases hepatic fat oxidation and reduces glucose synthesis, and also regulates the innate and adaptive immune system. In another study, total and LDL cholesterol was also lowered in hamsters fed Chardonnay GSE on low (5\%) fat diets ${ }^{[59]}$. Weight gain was not reported.

PA-rich GSE have been used almost exclusively in most nutritional studies. This is because PAs are the major bioactive component, they are readily extractable by water and/or alcohol, and their low concentration in grape seeds, $2 \%-3 \%$, would otherwise require high amounts of the whole seeds to be ingested in nutritional studies ${ }^{[15,60-62]}$. Also, pomace spoils rapidly. Extraction and purification of the PAs removes microbial contaminants, and the reduced number of constituents allows for the chemical definition of the extracts. However, extractable polyphenols are a minor component compared to the nonextractable residue $^{[17,63]}$ and the residue may contain useful levels of bioactive PAs.

Several nutritional studies have reported using a dietary fiber product from grape pomace containing from $15 \%$ to $30 \%$ nonextractable polyphenols and $1 \%$ to $9 \%$ extractable polyphenols ${ }^{[64,65]}$. In one study, ingredients from red grape peel (RGP), white grape peel (WGP) or white grape seed (WGS) were fed to rats on either a low fat (4\% soybean oil by weight) or high fat (15\% lard by weight) for 6 weeks ${ }^{[66]}$. The nonextractable polyphenol content of the RGP, WGP and WGS were $26.2 \%, 21.0 \%$ and $18.8 \%$, respectively. The extractable polyphenols for the RGP, WGP and WGS were $3.8 \%, 5.0 \%$ and $5.4 \%$, respectively. There were no differences in weight gain, feed intake or feed efficiency between any treatment with either low fat or high fat diets except for higher feed intake by the WGS on the high fat diet. All grape ingredients increased daily dry feces weight, and decreased protein and fat digestibility. All grape ingredients also lowered total, high density lipoprotein (HDL) and low density lipoprotein (LDL) cholesterol in rats fed the high fat diet. In a subsequent study of in vitro fermentability of white grape peel and seed, almost all of the extractable polyphenols were degraded. However, the same researchers had shown that condensed tannins in carob are not fermented in vitro, suggesting that they are not readily metabolized by gut bacteria ${ }^{[67]}$.

Although the use of extracts of grape seeds and skins is convenient and often necessary to remove microbial contaminants, the use of whole grape seeds and skins is attractive because their high dietary fiber content may act as a prebiotic and impact the microbiome. Freshly processed wine grape pomace is not easily sourced. The USDA laboratory in Albany collaborated with a Northern California winery that had a small plant to process varietal grape pomace into powdered skins and seeds ${ }^{[67-69]}$.

The seeds from three grape cultivars were fed to hamsters on high fat diets. The total flavonoid content of seeds were $12 \%, 8.6 \%$ and $6.3 \%$ for Chardonnay, Cabernet and Syrah, respectively. Total catechins were $1.6 \%, 0.25 \%$, and $0.19 \%$ for the Chardonnay, Cabernet, and Syrah seeds, respectively. The high fat diets $(20 \%$ fat by weight) were supplemented with either 10\% Chardonnay (ChrSd), Cabernet (CabSd), or Syrah grape seed (SyrSd). The macronutrient composition of the grape seed diets was adjusted by decreasing amounts of casein, corn starch, microcrystalline cellulose for the amounts in the grape seed. Energy intake was higher in hamsters fed SyrSd compared to the controls, 3.55 versus $3.14 \mathrm{MJ}$, 
respectively. Energy intake was not significantly different for the hamsters fed ChrSd and CabSd, 3.39 and $3.45 \mathrm{MJ}$, respectively. Despite the higher energy intake, weight gain was lowest with ChrdSd, compared to the controls, 23.9 versus $34.7 \mathrm{~g}$. Weight gains with $\mathrm{CabSd}$ and $\mathrm{SyrSd}$ were 38.8 and $40.5 \mathrm{~g}$, respectively. Plasma VLDL and LDL cholesterol were markedly lower in ChrSd hamsters, 6.5 and $33.4 \mathrm{mg} \cdot \mathrm{dL}^{-1}$, compared to 23.6 and $75.2 \mathrm{mg} \cdot \mathrm{dL}^{-1}$, respectively, in the controls (Fig.5). Adipose and liver weight were also significantly lower, $23 \%$ and $38 \%$, respectively, in with ChrSd compared to the controls. The expression of hepatic genes CYP51, CYP7A1 and LDLR was significantly correlated with changes in plasma total, VLDL or LDL cholesterol concentrations. Some important bacterial groups were analyzed in the feces by PCR methods: Bacteroides fragilis, Lactobacillus spp., Clostridium cluster IV, Enterococcus spp., Bifidobacterium spp., Enterobacteriaceae and total bacteria. The number of total bacteria was reduced from $\log 10.7$ to $\log 9.7^{[70]}$. Lactobacillus spp. were positively correlated with total and LDL cholesterol and liver weight. $B$. fragilis and Bifidobacterium spp. were negatively and positively correlated, respectively, with liver weight. The phylum Proteobacteria was positively correlated with VLDL cholesterol and adipose tissue weight. A link between intestinal bacteria and hepatic bile acid synthesis was also found. The expression of intestinal FGF15 was found to decrease in hamsters fed ChrdSd. The FGF15 product circulates to the liver where it downregulates hepatic CYP7A1. The FGF15 product represses CYP7A1 and decreasing intestinal synthesis would increase liver bile acid synthesis and reduce plasma cholesterol levels. $F G F 15$ is itself regulated by intestinal FXR, a nuclear receptor with bile acids as natural ligands. Bile acid levels depend on certain species of bacteria that can hydrolyze

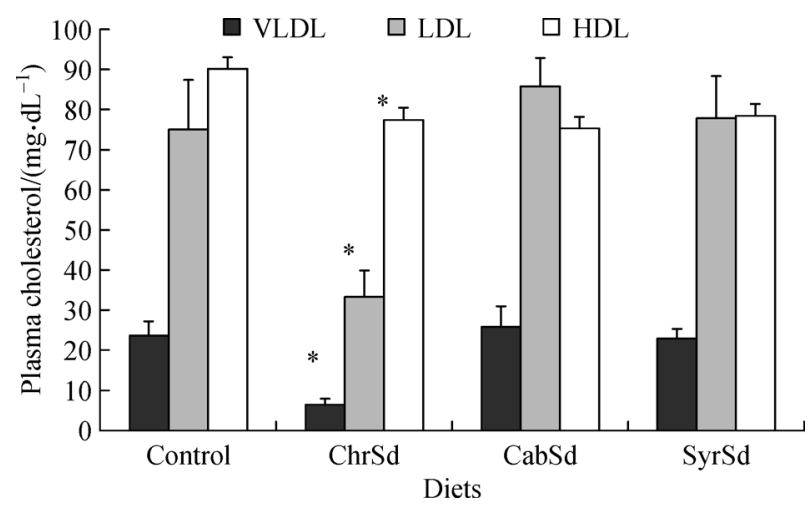

Fig. 5 Effect of powdered grape seeds on Syrian Hamster HDL, LDL and VDL. Male Syrian hamsters were fed high fat diets containing $10 \%$ of the powdered seeds of Chardonnay (ChrSd), Cabernet Sauvignon (CabSd), and Syrah (SyrSd) grapes for four weeks. High density lipoprotein (HDL), low density lipoprotein (LDL) and very low density lipoprotein (VLDL) cholesterol were lowered in hamsters fed the ChrSd diets compared to hamsters fed the Control. conjugated bile acids. Lactobacillus possesses bile salt hydrolase activity and the lower numbers of Lactobacillus would decrease deconjugation and increase levels of bile acids in the lumen. Bile acids are FXR antagonists ${ }^{[71]}$. While PAs are the most bioactive components of grape seeds, grape seed powder also contained large amounts of fermentable dietary fiber which may also change the composition of the gut microbiota and affect plasma cholesterol levels.

The number of human studies of grape seed PAs are limited. In 2011, Feringa et al. ${ }^{[72]}$ published a metaanalysis of human trials of grape seed extracts on cardiovascular risk markers. The analysis included studies indexed by Medline, Cochrane Central Registry of Clinical Trials, Agricola and Scopus up to 2010. Nine clinical trials were found to meet their selection criteria. There were no statistically significant change in total, LDL and HDL cholesterol, or triglycerides. There was a trend to lower total and LDL cholesterol and higher HDL cholesterol. However, there was a small but significant decrease in systolic blood pressure and heart rate. A more recent metaanalysis of human clinical trials of the effects of grape seed extract on blood pressure has been published ${ }^{[73]}$. The authors analyzed data from a search of PubMed and Embase through to November 2015 and found 12 relevant trials. Four of the trials reported low and high doses, and were treated separately, thus 16 trials were reported. Dose ranged from 100 to $2000 \mathrm{mg} \cdot \mathrm{d}^{-1}$ and included 840 subjects. The meta-analysis found that both systolic and diastolic pressures were lowered significantly ${ }^{[73]}$, supporting the earlier Feringa study ${ }^{[72]}$.

\section{Conclusions}

Obesity related metabolic diseases may be prevented, or their severity reduced, by the intake of grape PAs and other plant components high in phytochemicals. The decrease in the intake of astringent plant phenolic and dietary fibers when societies become more affluent may be related to obesity and diabetes increases. While animal studies consistently show reductions in weight gain, adipose weight gain, plasma and liver lipids, and liver weight and lipid content, human studies have not shown similar effects. PAs interact with the many proteins secreted by the salivary glands and different areas of the digestive system. The most bioactive forms appear to be the oligomeric procyanidins. In the gut, they modify the numbers of total bacteria and promote some groups at the expense of others. They are metabolized by gut bacteria to phenolic acids that are absorbed into the circulation of the host, however, it is not clear how procyanidins alter host metabolism to reduce obesity and risk factors for chronic diseases.

Acknowledgements This work was supported by USDA, ARS CRIS Project 2030-41440-007-00-D. 
Compliance with ethics guidelines Torey Arvik, Hyunsook Kim, James Seiber, and Wallace Yokoyama declare that they have no conflicts of interest or financial conflicts to disclose.

This article is a review and does not contain any studies with human or animal subjects performed by any of the authors.

\section{References}

1. Caputo T, Gilardi F, Desvergne B. From chronic overnutrition to metaflammation and insulin resistance: adipose tissue and liver contributions. FEBS Letters, 2017, 591(19): 3061-3088

2. Mancuso P. The role of adipokines in chronic inflammation. Immuno Targets and Therapy, 2016, 5: 47-56

3. Hamilton M K, Raybould H E. Bugs, guts and brains, and the regulation of food intake and body weight. International Journal of Molecular Sciences 2016, 6 (S1): 8-14

4. Kumari M, Kozyrskyj A L. Gut microbial metabolism defines host metabolism: an emerging perspective in obesity and allergic inflammation. Obesity Reviews: an Official Journal of the International Association for the Study of Obesity, 2017, 18(1): 18-31

5. Wen L, Duffy A. Factors influencing the gut microbiota, inflammation, and type 2 diabetes. Journal of Nutritional Biochemistry, 2017, 147(7): 1468S-1475S

6. Hruby A, Hu F B. The epidemiology of obesity: a big picture. PharmacoEconomics, 2015, 33(7): 673-689

7. Peng W, Berry E M. Global nutrition 1990-2015: a shrinking hungry, and expanding fat world. PLoS One, 2018, 13(3): e0194821

8. Araújo J R, Tomas J, Brenner C, Sansonetti P J. Impact of high-fat diet on the intestinal microbiota and small intestinal physiology before and after the onset of obesity. Biochimie, 2017, 141: 97-106

9. King H, Aubert R E, Herman W H. Global burden of diabetes, 1995-2025: prevalence, numerical estimates, and projections. Diabetes Care, 1998, 21(9): 1414-1431

10. NCD Risk Factor Collaboration (NCD-RisC). Trends in adult bodymass index in 200 countries from 1975 to 2014: a pooled analysis of 1698 population-based measurement studies with 19.2 million participants. Lancet, 2016, 387(10026): 1377-1396

11. Wang H J, Zhang B, Du W W, Liu A D, Zhang J G, Wang Z H, Su C, Ma Y X, Zhai F Y. Trends of the dietary fiber intake among Chinese aged 18-45 in nine provinces (autonomous region) from 1989 to 2006. Chinese Journal of Preventive Medicine, 2011, 45(4): 318322

12. Xiao Y, Su C, Ouyang Y, Zhang B. Trends of vegetables and fruits consumption among Chinese adults aged 18 to 44 years old from 1991 to 2011. Chinese Journal of Epidemiology, 2015, 36(3): 232236

13. Ngamukote S, Mäkynen $\mathrm{K}$, Thilawech $\mathrm{T}$, Adisakwattana $\mathrm{S}$. Cholesterol-lowering activity of the major polyphenols in grape seed. Molecules, 2011, 16(6): 5054-5061

14. Prior $\mathrm{R} \mathrm{L}, \mathrm{Gu} \mathrm{L}$. Occurrence and biological significance of proanthocyanidins in the American diet. Phytochemistry, 2005, 66 (18): 2264-2280

15. Fuleki T, Ricardo-da-Silva J M. Catechin and procyanidin composition of seeds from grape cultivars grown in Ontario. Journal of Agricultural and Food Chemistry, 1997, 45(4): 11561160
16. Arranz S, Saura-Calixto F, Shaha S, Kroon P A. High contents of nonextractable polyphenols in fruits suggest that polyphenol contents of plant foods have been underestimated. Journal of Agricultural and Food Chemistry, 2009, 57(16): 7298-7303

17. Perez-Jimenez J, Arranz S, Saura-Calixto F. Proanthocyanidin content in foods is largely underestimated in the literature data: an approach to quantification of the missing proanthocyanidins. Food Research International, 2009, 42(10): 1381-1388

18. Weber H A, Hodges A E, Guthrie J R, O'Brien B M, Robaugh D, Clark A P, Harris R K, Algaier J W, Smith C S. Comparison of proanthocyanidins in commercial antioxidants: grape seed and pine bark extracts. Journal of Agricultural and Food Chemistry, 2007, 55 (1): 148-156

19. Cadot Y, Miñana-Castelló M T, Chevalier M. Anatomical, histological, and histochemical changes in grape seeds from Vitis vinifera $\mathrm{L}$. cv Cabernet franc during fruit development. Journal of Agricultural and Food Chemistry, 2006, 54(24): 9206-9215

20. Kennedy J A, Matthews M A, Waterhouse A L. Changes in grape seed polyphenols during fruit ripening. Phytochemistry, 2000, 55 (1): 77-85

21. Kennedy J A, Ristic R, Iland P G, Jones G P, Troup G J, Pilbrow J R, Hutton D R, Hewitt D, Hunter C R. Development of seed polyphenols in berries from Vitis vinifera L. cv. Shiraz. Australian Journal of Grape and Wine Research, 2000, 6(3): 244-254

22. del Rio J L P, Kennedy J A. Development of proanthocyanidins in Vitis vinifera L. cv. Pinot noir grapes and extraction into wine. American Journal of Enology and Viticulture, 2006, 57(2): 125-132

23. Moran M A, Sadras V O, Liebich B, Bubner R M. High temp traits differ across varieties. Australian \& New Zealand Grapegrower \& Winemaker, 2011, 571: 34-35

24. Cala O, Dufourc E J, Fouquet E, Manigand C, Laguerre M, Pianet I. The colloidal state of tannins impacts the nature of their interaction with proteins: the case of salivary proline-rich protein/procyanidins binding. Langmuir: the ACS Journal of Surfaces and Colloids, 2012, 28(50): 17410-17418

25. de Freitas V, Mateus N. Structural features of procyanidin interactions with salivary proteins. Journal of Agricultural and Food Chemistry, 2001, 49(2): 940-945

26. Carvalho E, Mateus N, Plet B, Pianet I, Dufourc E, De Freitas V. Influence of wine pectic polysaccharides on the interactions between condensed tannins and salivary proteins. Journal of Agricultural and Food Chemistry, 2006, 54(23): 8936-8944

27. Soares S, Mateus N, de Freitas V. Carbohydrates inhibit salivary proteins precipitation by condensed tannins. Journal of Agricultural and Food Chemistry, 2012, 60(15): 3966-3972

28. Gonçalves R, Mateus N, De Freitas V. Influence of carbohydrates on the interaction of procyanidin B3 with trypsin. Journal of Agricultural and Food Chemistry, 2011, 59(21): 11794-11802

29. Shirakami Y, Sakai H, Kochi T, Seishima M, Shimizu M. Catechins and its role in chronic diseases. Advances in Experimental Medicine and Biology, 2016, 929: 67-90

30. Zhang L, Wang Y, Li D, Ho C T, Li J, Wan X. The absorption, distribution, metabolism and excretion of procyanidins. Food \& Function, 2016, 7(3): 1273-1281

31. Glick Z, Joslyn M A. Effect of tannic acid and related compounds on the absorption and utilization of proteins in the rat. Journal of 
Nutrition, 1970, 100(5): 516-520

32. Oh H I, Hoff J E. Interaction of condensed grape tannins with pepsin and trypsin in simulated human digestive-system. Nutrition Reports International, 1988, 38(3): 445-453

33. Bates J M, Akerlund J, Mittge E, Guillemin K. Intestinal alkaline phosphatase detoxifies lipopolysaccharide and prevents inflammation in zebrafish in response to the gut microbiota. Cell Host \& Microbe, 2007, 2(6): 371-382

34. Estaki M, DeCoffe D, Gibson D L. Interplay between intestinal alkaline phosphatase, diet, gut microbes and immunity. World Journal of Gastroenterology, 2014, 20(42): 15650-15656

35. Malo M S, Alam S N, Mostafa G, Zeller S J, Johnson P V, Mohammad N, Chen K T, Moss A K, Ramasamy S, Faruqui A, Hodin S, Malo P S, Ebrahimi F, Biswas B, Narisawa S, Millán J L, Warren H S, Kaplan J B, Kitts C L, Hohmann E L, Hodin R A. Intestinal alkaline phosphatase preserves the normal homeostasis of gut microbiota. Gut, 2010, 59(11): 1476-1484

36. Tebib K, Rouanet J M, Besançon P. Effect of grape seed tannins on the activity of some rat intestinal enzyme activities. Enzyme Protein, 1994, 48(1): 51-60

37. Vallet J, Rouanet J M, Besançon P. Dietary grape seed tannins: effects of nutritional balance and on some enzymic activities along the crypt-villus axis of rat small intestine. Annals of Nutrition \& Metabolism, 1994, 38(2): 75-84

38. Kaliannan K, Hamarneh S R, Economopoulos K P, Nasrin Alam S, Moaven O, Patel P, Malo N S, Ray M, Abtahi S M, Muhammad N, Raychowdhury A, Teshager A, Mohamed M M, Moss A K, Ahmed R, Hakimian S, Narisawa S, Millán J L, Hohmann E, Warren H S, Bhan A K, Malo M S, Hodin R A. Intestinal alkaline phosphatase prevents metabolic syndrome in mice. Proceedings of the National Academy of Sciences of the United States of America, 2013, 110(17): 7003-7008

39. Sugiyama H, Akazome Y, Shoji T, Yamaguchi A, Yasue M, Kanda $\mathrm{T}$, Ohtake Y. Oligomeric procyanidins in apple polyphenol are main active components for inhibition of pancreatic lipase and triglyceride absorption. Journal of Agricultural and Food Chemistry, 2007, 55(11): 4604-4609

40. Moreno D A, Ilic N, Poulev A, Brasaemle D L, Fried S K, Raskin I. Inhibitory effects of grape seed extract on lipases. Nutrition, 2003, 19(10): 876-879

41. Adisakwattana S, Moonrat J, Srichairat S, Chanasit C, Tirapongporn H, Chanathong B, Ngamukote S, Makynen K, Sapwarobol S. Lowering mechanisms of grape seed extract (Vitis vinifera $\mathrm{L}$ ) and its antihyperlidemic activity. Journal of Medicinal Plants Research, 2010, 4(20): 2113-2120

42. Donovan J L, Manach C, Rios L, Morand C, Scalbert A, Rémésy C. Procyanidins are not bioavailable in rats fed a single meal containing a grapeseed extract or the procyanidin dimer B3. British Journal of Nutrition, 2002, 87(4): 299-306

43. Baba S, Osakabe N, Natsume M, Terao J. Absorption and urinary excretion of procyanidin B2 [epicatechin-(4 $\beta-8)$-epicatechin] in rats. Free Radical Biology \& Medicine, 2002, 33(1): 142-148

44. Choy Y Y, Jaggers G K, Oteiza P I, Waterhouse A L. Bioavailability of intact proanthocyanidins in the rat colon after ingestion of grape seed extract. Journal of Agricultural and Food Chemistry, 2013, 61 (1): $121-127$
45. Choy Y Y, Quifer-Rada P, Holstege D M, Frese S A, Calvert C C, Mills D A, Lamuela-Raventos R M, Waterhouse A L. Phenolic metabolites and substantial microbiome changes in pig feces by ingesting grape seed proanthocyanidins. Food \& Function, 2014, 5 (9): 2298-2308

46. Liu W, Zhao S, Wang J, Shi J, Sun Y, Wang W, Ning G, Hong J, Liu R. Molecular Nutrition \& Food Research, 2017, 61(9): 1601082

47. Yokota A, Fukiya S, Islam K B, Ooka T, Ogura Y, Hayashi T, Hagio $\mathrm{M}$, Ishizuka S. Is bile acid a determinant of the gut microbiota on a high-fat diet? Gut Microbes, 2012, 3(5): 455-459

48. Tamura T, Ozawa M, Tanaka N, Arai S, Mura K. Bacillus cereus response to a proanthocyanidin trimer, a transcriptional and functional analysis. Current Microbiology, 2016, 73(1): 115-123

49. Tebib K, Bitri L, Besancon P, Rouanet J. Polymeric grape seed tannins prevent plasma cholesterol changes in high-cholesterol-fed rats. Food Chemistry, 1994, 49(4): 403-406

50. Martin-Carron N, Goni I, Larrauri J A, Garcia-Alonso A, SauraCalixto F. Reduction in serum total and LDL cholesterol concentrations by a dietary fiber and polyphenol-rich grape product in hypercholesterolemic rats. Nutrition Research, 1999, 19(9): 1371-1381

51. Nakamura Y, Tonogai Y. Effects of grape seed polyphenols on serum and hepatic lipid contents and fecal steroid excretion in normal and hypercholesterolemic rats. Journal of Health Science, 2002, 48(6): 570-578

52. Bagchi D, Bagchi M, Stohs S J, Das D K, Ray S D, Kuszynski C A, Joshi S S, Pruess H G. Free radicals and grape seed proanthocyanidin extract: importance in human health and disease prevention. Toxicology, 2000, 148(2-3): 187-197

53. Bagchi D, Bagchi M, Stohs S, Ray S D, Sen C K, Preuss H G. Cellular protection with proanthocyanidins derived from grape seeds. Annals of the New York Academy of Sciences, 2002, 957(1): 260-270

54. Bagchi D, Swaroop A, Preuss H G, Bagchi M. Free radical scavenging, antioxidant and cancer chemoprevention by grape seed proanthocyanidin: an overview. Mutation Research, 2014, 768: 6973

55. Décordé K, Teissèdre P L, Sutra T, Ventura E, Cristol J P, Rouanet J M. Chardonnay grape seed procyanidin extract supplementation prevents high-fat diet-induced obesity in hamsters by improving adipokine imbalance and oxidative stress markers. Molecular Nutrition \& Food Research, 2009, 53(5): 659-666

56. Zhang Z, Wang H, Jiao R, Peng C, Wong Y M, Yeung V S, Huang Y, Chen Z Y. Choosing hamsters but not rats as a model for studying plasma cholesterol-lowering activity of functional foods. Molecular Nutrition \& Food Research, 2009, 53(7): 921-930

57. Cao H. Adipocytokines in obesity and metabolic disease. Journal of Endocrinology, 2014, 220(2): T47-T59

58. Andrade-Oliveira V, Camara N O, Moraes-Vieira P M. Adipokines as drug targets in diabetes and underlying disturbances. Journal of Diabetes Research, 2015, 2015: 681612

59. Jiao R, Zhang Z, Yu H, Huang Y, Chen Z Y. Hypocholesterolemic activity of grape seed proanthocyanidin is mediated by enhancement of bile acid excretion and up-regulation of CYP7A1. Journal of Nutritional Biochemistry, 2010, 21(11): 1134-1139

60. Bucic-Kojic A, Planinic M, Tomas S, Jakobek L, Seruga M. 
Influence of solvent and temperature on extraction of phenolic compounds from grape seed, antioxidant activity and colour of extract. International Journal of Food Science \& Technology, 2009, 44(12): 2394-2401

61. Bautista-Ortin A B, Rodriguez-Rodriguez P, Gil-Munoz R, Jimenez-Pascual E, Busse-Valverde N, Martinez-Cutillas A, Lopez-Roca J M, Gomez-Plaza E. Influence of berry ripeness on concentration, qualitative composition and extractability of grape seed tannins. Australian Journal of Grape and Wine Research, 2012, 18(2): 123-130

62. Cádiz-Gurrea M L, Borrás-Linares I, Lozano-Sánchez J, Joven J, Fernández-Arroyo S, Segura-Carretero A. Cocoa and grape seed byproducts as a source of antioxidant and anti-inflammatory proanthocyanidins. International Journal of Molecular Sciences, 2017, 18(2): E376

63. Hellström J K, Törrönen A R, Mattila P H. Proanthocyanidins in common food products of plant origin. Journal of Agricultural and Food Chemistry, 2009, 57(17): 7899-7906

64. Saura-Calixto F. Antioxidant dietary fiber product: a new concept and a potential food ingredient. Journal of Agricultural and Food Chemistry, 1998, 46(10): 4303-4306

65. Bravo L, Saura-Calixto F. Characterization of dietary fiber and the in vitro indigestible fraction of grape pomace. American Journal of Enology and Viticulture, 1998, 49(2): 135-141

66. Martin-Carron N, Saura-Calixto F, Goni I. Effects of dietary fibreand polyphenol-rich grape products on lipidaemia and nutritional parameters in rats. Journal of the Science of Food and Agriculture, 2000, 80(8): 1183-1188

67. Bravo L, Abia R, Sauracalixto F. Polyphenols as dietary fiber associated compounds - Comparative-study in-vivo and in-vitro properties. Journal of Agricultural and Food Chemistry, 1994, 42 (7): 1481-1487

68. Kim H, Bartley G, Chon J W, Seo K H, Yokoyama W. Chardonnay grape seed flour alters expression of hepatic genes for lipid and glucose metabolism, inflammation, and leptin receptor. FASEB Journal, 2014, 28(1)

69. Seo K, Kim H, Chon J, Kim D, Nah S, Arvik T, Yokoyama W. Flavonoid-rich Chardonnay grape seed flour supplementation ameliorates diet-induced visceral adiposity, insulin resistance, and glucose intolerance via altered adipose tissue gene expression. Journal of Functional Foods, 2015, 17: 881-891

70. Kim H, Kim D H, Seo K H, Chon J W, Nah S Y, Bartley G E, Arvik $\mathrm{T}$, Lipson R, Yokoyama W. Modulation of the intestinal microbiota is associated with lower plasma cholesterol and weight gain in hamsters fed Chardonnay grape seed flour. Journal of Agricultural and Food Chemistry, 2015, 63(5): 1460-1467

71. Li F, Jiang C, Krausz K W, Li Y, Albert I, Hao H, Fabre K M, Mitchell J B, Patterson A D, Gonzalez F J. Microbiome remodelling leads to inhibition of intestinal farnesoid $\mathrm{X}$ receptor signalling and decreased obesity. Nature Communications, 2013, 4(1): 2384

72. Feringa H H, Laskey D A, Dickson J E, Coleman C I. The effect of grape seed extract on cardiovascular risk markers: a meta-analysis of randomized controlled trials. Journal of the American Dietetic Association, 2011, 111(8): 1173-1181

73. Zhang H, Liu S, Li L, Liu S, Liu S, Mi J, Tian G. The impact of grape seed extract treatment on blood pressure changes: a metaanalysis of 16 randomized controlled trials. Medicine, 2016, 95(33): e4247 